\title{
Medical Cytogenetics
}

\section{Reviewed by Shinichi Misawa}

\author{
Department of Medicine, Kyoto Prefectural Yosanoumi Hospital, Iwatakicho, Japan
}

Int J Hematol. 2001;73:266.

(C)2001 The Japanese Society of Hematology

\begin{abstract}
Medical Cytogenetics, edited by H. F. L. Mark. New York: Marcel Dekker, 2000; 680 pages.

Some time ago it was believed that chromosomal analysis had the best potential for molecular understanding of cancer and leukemia. The rapid advancement of powerful tools for the study of molecular genetics seems to have terminated the major role of cytogenetics. Under the circumstances, one might wonder about the usefulness of a textbook of cytogenetics. Cytogenetics, however, has continued to advance in technology and knowledge at a pace comparable to molecular genetics.
\end{abstract}

Few books give an overview of medical genetics and clinical cytogenetics, although there are numerous reference books and technical texts on cytogenetics. Medical Cytogenetics provides complete coverage of many current applications of cytogenetics in medicine. It is edited by Hon Fong L. Mark, with individual chapters written by distinguished authors, many of them are top experts in their fields. These authors describe this rapidly evolving field in a simple and comprehensible fashion giving many examples and illustrations.

The target audience of this book will be medical practitioners, including pathologists, internists, hematologists, medical oncologists, pediatricians, obstetricians, and other specialists. Scientists who desire a review of the field of cytogenetics, technicians in training or in practice, and medical or graduate students interested in medical genetics will find this publication to be a useful reference on cytogenetics.

The volume consists of 20 chapters. The first chapter includes a concise history of cytogenetics, which begins with the statement of Mendel's laws proposed in 1865. The authors also briefly discuss the Board of Medical Genetics in the United States. Chapters 2 through 6 cover basic cytogenetic information about the human genome and illustrate culturing and staining techniques. Human cytogenetic nomenclature is also described in detail, and a thorough introduc- tion to the technique of fluorescence in situ hybridization (FISH) is provided. Chapter 7 introduces the application of cytogenetics in medicine. Later chapters focus on more specialized topics in cytogenetics: prenatal chromosomal diagnosis and genetic counseling; cytogenetics of reproductive wastage; cytogenetics of male infertility; cytogenetics of leukemia, lymphoma, and solid tumors; and chromosomal instability and fragile sites. Cytogenetics of transfusion medicine, discussed in Chapter 16, is a unique issue not usually covered in cytogenetics texts. The last few chapters are devoted to perspectives on the future of cytogenetics. The new techniques of comparative genomic hybridization and spectral karyotyping (SKY) are introduced and are expected to become routine techniques for the average clinical cytogenetics laboratory. Quality control and quality assurance are defined and provide a reference for setting standards in cytogenetics laboratories.

The format of this book is well suited to help readers grasp basic cytogenetic concepts. Each chapter includes a glossary of terms, which ensures clear understanding of the factual material, and a current bibliography for those who want to read the original sources. At the end of the book are 5 to 28 practice questions for each chapter. Answers to these questions depend on one's level of knowledge and technical achievement in cytogenetics.

Medical Cytogenetics thus provides a concise and comprehensive overview of the field of cytogenetics in medicine. This volume is highly recommended for experienced medical practitioners and medical cytogeneticists and trainees, and would be a good addition to institutional and personal libraries.

Finally, when you first open the book, note an impressive sentence in the Acknowledgments. Dr. Mark writes that 2 of her sons died of a rare genetic disease contracted from their parents, which has been one of the motivations for Dr. Mark to advance in her specialty. It is in that sense that her editing of this book is in memory of her sons. 